\title{
Stabilometry platform benefits in diagnosis and occlusal orthosis treatment of occluso-postural deficiency: preliminary study on 30 patients
}

\author{
Jean-Claude Combadazou ${ }^{1}$, Antonin Hennequin ${ }^{1}$, Mathieu Benichou ${ }^{2}$, Capucine Roumiguié2 and Florent Destruhaut $^{1 *}$ \\ ${ }^{1}$ Faculty of Odontology, University Toulouse III, Rangueil Hospital, France \\ ${ }^{2}$ Rangueil Hospital, Toulouse, France
}

\begin{abstract}
Objectives: The primary aim of this work was to determine if using of a stabilometry platform in a postural deficiency diagnosis with the influence of an occlusal sensor was valuable. A secondary aim was to determine the impact of the orthosis occlusal treatment based on a record from a stabilometry platform.

Methods and materials: Thirty adults with temporomandibular disorders (TDM) and postural pain were included in this study. Statokinesigrams and stabilograms recordings were realized on patients before and after occlusal device treatment.

Results: Comparison of data plots (statokinesigram and stabilogram) before and after treatment is only significant for one of the five main postural values extracted from stabilometric standard parameters: the sustentation polygon's area. The treatment impact on this value is moderated.

Conclusion: The use of a stabilometric platform in the study of the influence of an occlusal device treatment is interesting, especially by analyzing the sustentation polygon's area for each patient (before and after occlusal treatment).
\end{abstract}

\section{Introduction}

The posture can be defined as edifying and active in the maintaining of the different body parts in the space and translates the way the body challenges different situations and reacts toward the outside world. The posture is the convergence of muscular activity (tonic and phasic) and is related to a cranio-cervico-mandibular balance. On an anthropologic plan, postural stability is a flexible concept due to many ways for a human being to maintain around his balanced position. The posture aims to allow the movement of a body segment whereas it stabilizes the others segments and insures the maintaining of the erected station and fights against gravity. Regarding the literature there is no real consensus on a link between dental occlusion and posture when using measuring instrument to evaluate it. The different studies are contradictory. During the Milan consensus conference in 1997, it has been reported that there was no scientific proof to support a link between occlusion and posture [1]. Perinetti in 2006, has shown on a 26 patient's cohort, with the help of posturography, the lack of postural modifications when the occlusion was changing [2]. Mandredini, et al. [3] has demonstrated throughout a systematic review that different techniques and posturoraphic devices failed to detect an association between body posture and dental occlusion. In some cases a link was detected, but it couldn't be statistically significant [4]. The most complete evaluation published concludes that the utility of instruments such as surface electromyography, kinesigraphy, stabilometry platform and other postural devices is still weak on dentistry techniques [5].

Those previous results seems to be in contradiction with other studies such as Bracco, et al. [6] and recently Alwarawreh, et al. [7]. The authors concluded on a 952 cohort that a real link between malocclusion severity and impact on the frontal plan of the posture on young adult males was statistically significant [7]. Many research work correlated to case reports tend to show a real link between occlusion and posture but this relation is still today hard to measure with the help of postural devices [6-13].

With that in mind, the primary aim of this work was to determine if the use of a stabilometric platform was useful in the diagnosis of a postural deficiency correlated to malocclusion. A secondary aim was to determine the impact of an orthosis occlusal treatment on the data plots extracted from the records of the stabilometric platform.

\section{Methods and materials}

The study was conducted in compliance with the Declaration of Helsinki, and informed consent was obtained from each subject.

\section{Selection criteria}

Thirty subjects were included in this study: there were all referred from occlusodonty consultation by posturologists and osteopaths because they presented postural pain. They all had an improvement

*Correspondence to: Florent Destruhaut, Faculté de Chirurgie Dentaire, 3 chemin des Maraichers 31400 TOULOUSE, France, Tel: +33 (0)6 748334 59; E-mail: destruhautflorent@yahoo.fr

Key words: temporomandibular disorders, neuromuscular occlusodontology, stabilometry platform

Received: September 17, 2019; Accepted: October 03, 2019; Published: October 07,2019 
of this pain after an orthosis occlusal treatment. This improvement is collected with the help of the Von Korff scale before and after treatment [14-16] (Figure 1). Each (consecutive) patient answered a medical questionnaire and underwent a clinical examination. Specific clinical signs were investigated, including pain on palpation of the masseter and temporal muscles, asynchronous contraction of the masseter muscles, temporomandibular joint sounds (crackling, cracking, clicking), and mouth opening limitation.

Adult subjects with postural deficiency syndrome were included if they had at least one of the following clinical presentations: orofacial pain upon palpation of the masseter and/or temporal muscles, TMJ dysfunction, or limitation of mouth opening or reducible/non-reducible disc dislocation [17]. Every patient felt a clinical improvement after wearing an occlusal orthosis. This clinical improvement is objectified with the help of the Von Korff scale.

Patients were not included if they met one or more of the following criteria: having a pacemaker or other electrical device, systemic diseases, history of local or general trauma, neurological or psychiatric disorders, muscular diseases, bruxism, assumption of antiinflammatory, analgesic, antidepressant or muscle relaxant drugs, fixed or removable prostheses, fixed restorations that could affect occlusal surfaces, previous or concurrent orthodontic treatment or orthognathic surgery, and excessive facial hair, which would interfere with correct surface electromyographic recording procedure.

\section{Recordings}

Occlusal troubles were diagnosed by a clinical exam and with the help of recordings made by the K7 System by Myotronics ${ }^{\circledR}$ while allowing to simultaneous record mandibular movements and masticatory muscles activity (Figure 2). The EMG recordings and muscle activity were expressed as the root mean square of the amplitude, expressed in $\mu \mathrm{V}$. Two dentists (FD, JCC), who trained beforehand to ensure good reproducibility, made the EMG recordings (Figure 3).

Statokinesigram and stabilogram records were realized with the normalized SATEL platform. Records were realized between 2 months and a half and 9 months after being treated by an occlusal orthosis. The average time of records was 5 months. If the patient was also treated foot orthosis, they kept it while the records were done. The records were realized with the eyes closed. A record was made on the mandibular position (physiological resting position), in occlusion, and in occlusion on the orthosis. For each subject, we measured 5 main parameters of stabilometric standards parameters [18].

Those parameters were:

- The sustentation polygon's area,

- The average $\mathrm{X}$, which is the pressure center's average position regarding the medio-lateral axes,

- The VfY, relation between the gravity center speed movement and the average movement on the $\mathrm{Y}$ axe antero-posterior),

- The LFS (comparison of length of movement and covered area),

- The Ratio AmpX/AmpY (ratio of the amplitude on the mediolateral axes and the antero-posterior axes) (Figure 4).

\section{Statistics}

In this the study, we compared the results of an orthosis treatment on each person. To achieve this, we are using the Wilcoxon signed rank
What number best describes your plain on average in the past week?

$\begin{array}{ccccccccccc}\begin{array}{c}0 \\ \text { (No Pain) }\end{array} & 1 & 2 & 3 & 4 & 5 & 6 & 7 & 8 & \begin{array}{c}9 \\ \text { (Pain as bad as } \\ \text { you can imagine) }\end{array}\end{array}$

What number best describes how, during the past week, pain has intertere with your general activity?

$\begin{array}{cccccccccc}0 & 1 & 2 & 3 & 4 & 5 & 6 & 7 & 8 & 9 \begin{array}{c}10 \\ \text { (Noain as bad as } \\ \text { you can imagine) }\end{array}\end{array}$

What number best describes how, during the past week, pain has intertered with your enjoyment in lite?

$\begin{array}{cccccccccc}\begin{array}{c}0 \\ \text { (No Pain) }\end{array} & 1 & 2 & 3 & 4 & 5 & 6 & 7 & 8 & \begin{array}{c}9 \\ \text { (Pain as bad as } \\ \text { you can imagine) }\end{array}\end{array}$

Figure 1. Von Korff scale

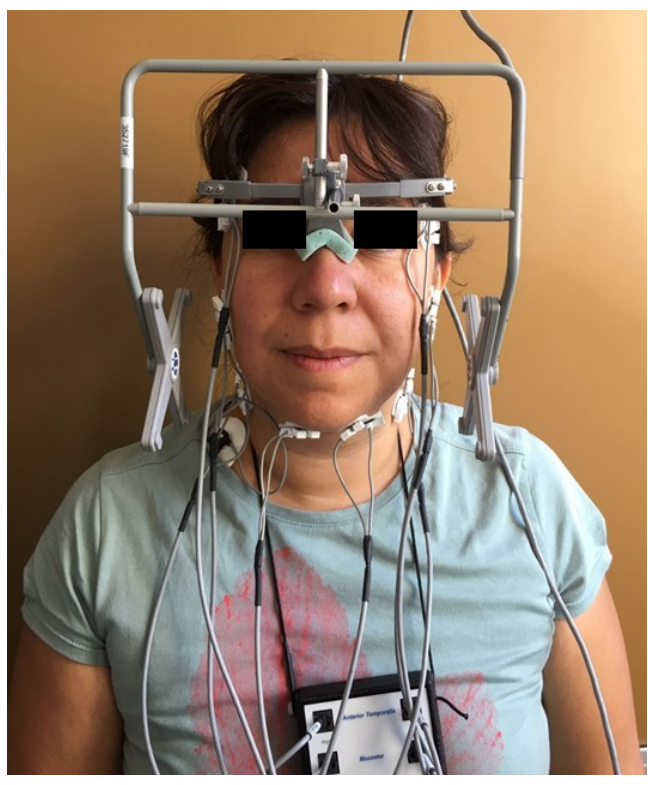

Figure 2. K7 System by Myotronics ${ }^{\circledR}$ on a patient

test. This test will allow us to compare two measures of a quantitative variable on the same subject. It will take any value, rank it in a number sequence, for each, a «stamp » with the before and after value will be attributed. We will have a significant results if the distributions are offset. The significance level is set to 0.05 . The Wilcoxon test is made throughout he five following parameters : the sustentation polygon's area, the average $\mathrm{X}$, the VfY, the LFS, and the AmpX/AmpY Ratio.

In a second time, calculating the Cohen's D will allow us to calculate the size of the effect (Figure 5). We will know the orthosis effect on stabilometry parameters considered significant by the Wilcoxon test. Cohen considers that the impact is weak for a $d=0.2$, average for $\mathrm{a}=$ 0.5 and strong for a $\mathrm{d}=0.8$. 

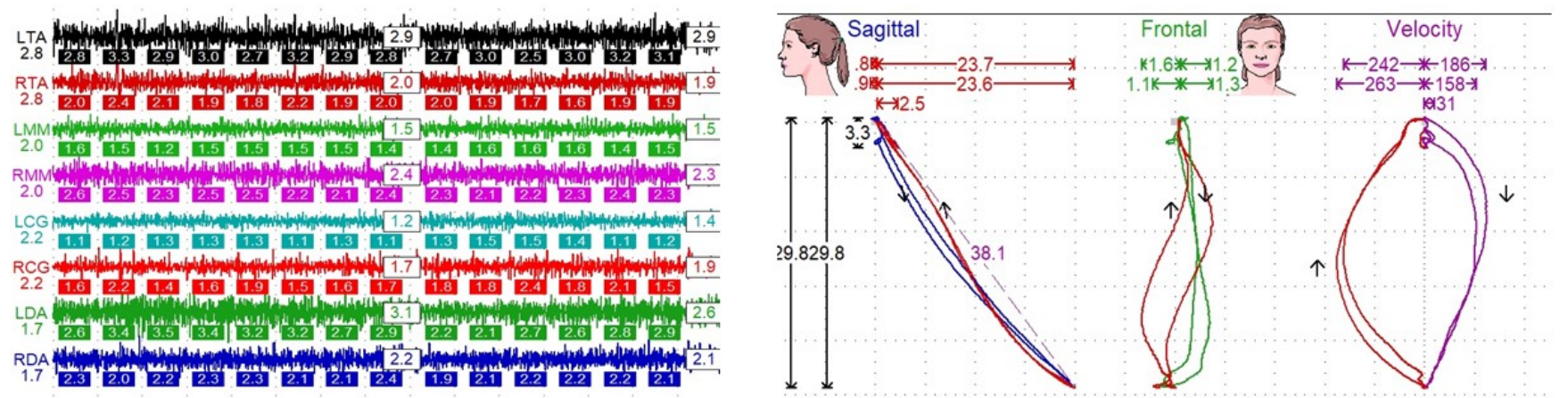

Time : $\quad \begin{array}{llllllllllllll}0: 01 & 0: 02 & 0: 03 & 0: 04 & 0: 05 & 0: 06 & 0: 07 & 0: 08 & 0: 09 & 0: 10 & 0: 11 & 0: 12 & 0: 13 & 0: 14\end{array}$
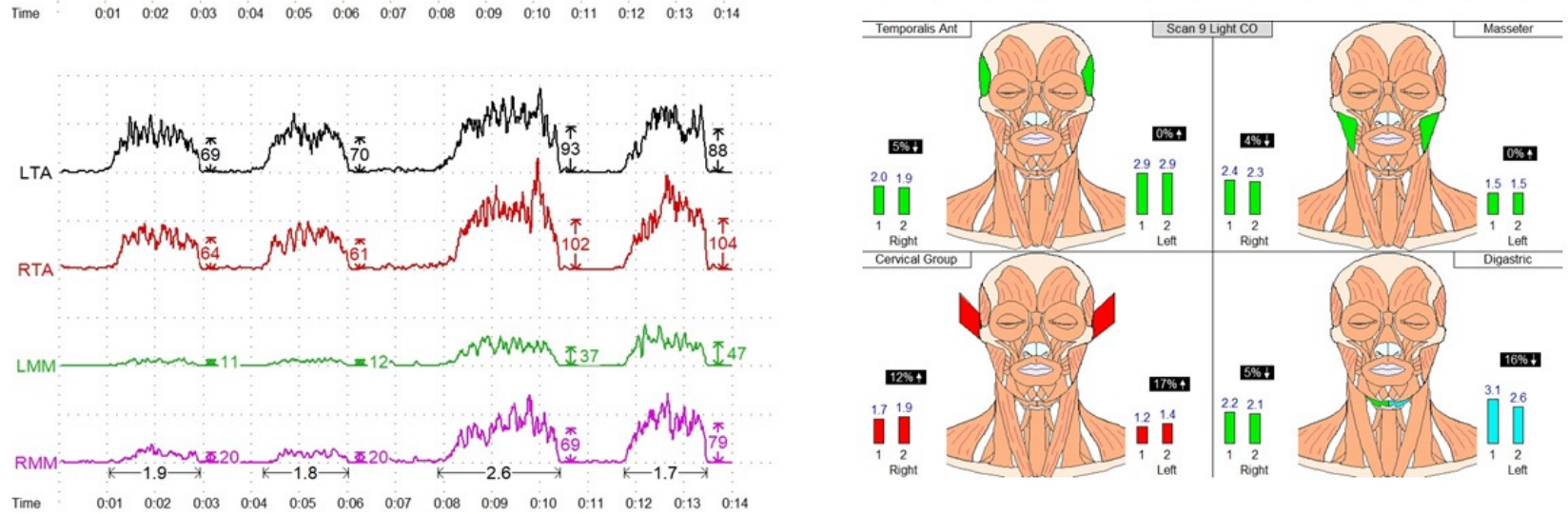

Figure 3. Electromyography recordings, Farrar's diagram and velocity-time graph (example)

Durée $(\mathrm{s}): 51.20$

Fréq. $(\mathrm{Hz}): 5$

Yeux Ouverts

Surface $\left(\mathrm{mm}^{2}\right)$

Longueur $X Y(\mathrm{~mm})$

LFS

VFY

$X$ moyen $(\mathrm{mm})$

$Y$ moyen $(\mathrm{mm})$

E. type $X(\mathrm{~mm})$

E. type $Y(\mathrm{~mm})$

Vitesse moy $(\mathrm{mm} / \mathrm{s})$

Var. vit. $(\mathrm{mm} / \mathrm{s})$

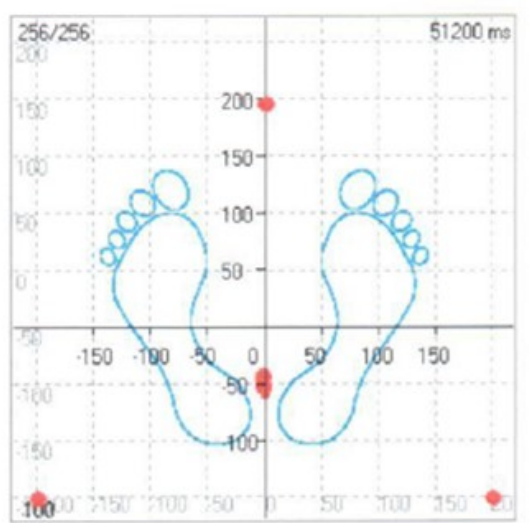

$:(39.00>91.00<210.00) \quad 167.4$

$:(307.00>429.00<599.00) \quad 412.5$

$(0.72>1.00<1.39)$

$(-2.61>0.00<3.59)$

$(-10.00>1.00<12.00)$

$(-57.00>-29.00<-1.00)$

$(3.00>10.00<18.00)$
0.45

$-1.77$

$-0.6$

$-51.5$

2.2

5.2

8.1

14.0

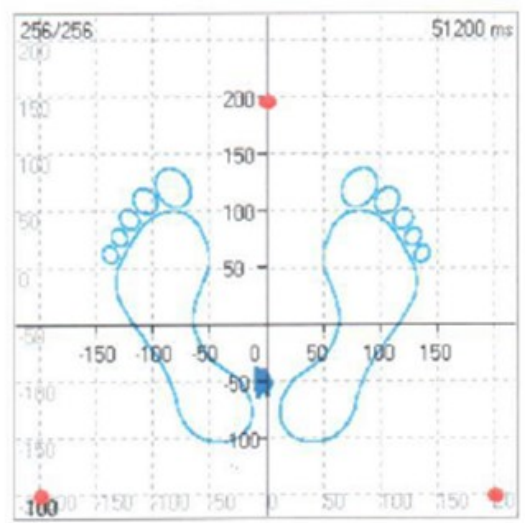

155.1

Figure 4. Statokinesigram recordings (example)

$(-4.73>0.00<4.86)$

$(-10.00>0.00<11.00)$

$(-51.00>-27.00<-3.00)$

-3.8
-51.8

2.7

4.0

9.8

17.7

$(5.00>11.00<18.00)$
$(79.00>225.00<638.00)$

$(346.00>613.00<880.00)$

Quotient B/A

92.65

122.00

Durée (s) : 51.20

Fréq. $(\mathrm{Hz}): 5$

Yeux Fermés 

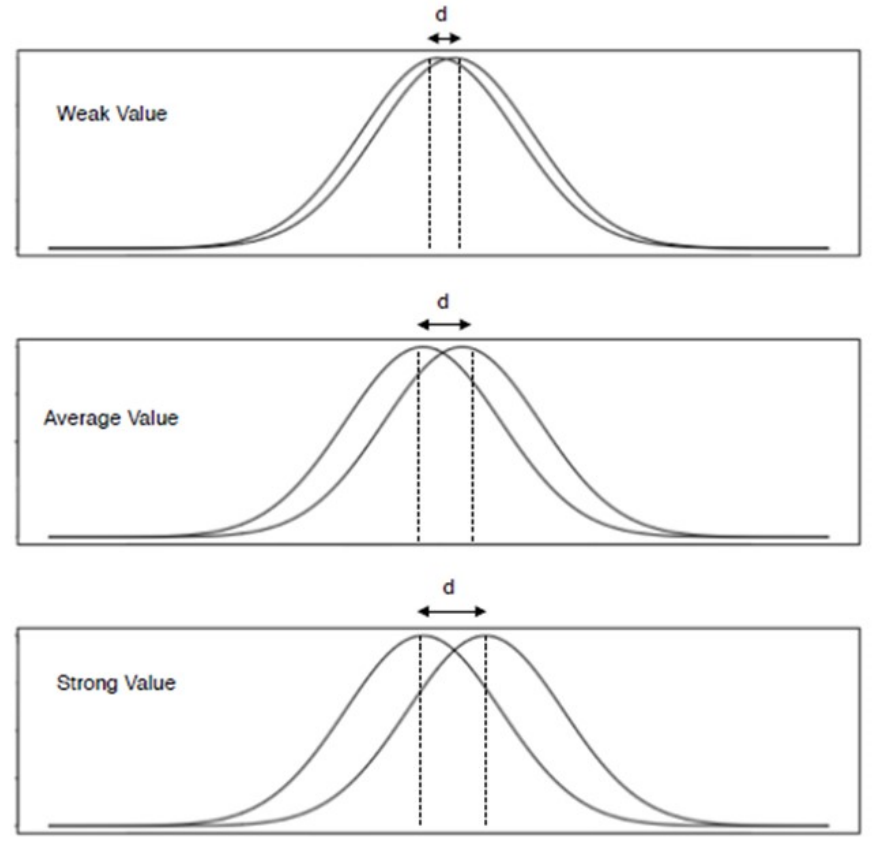

Figure 5. Cohen's d Value

\section{Results}

The Wilocoxon test's results are obtain in two stages: mandibular position (physiological rest position) and « teeth in occlusion» on the orthosis.

\section{Wilcoxon test's results in mandibular position}

The test will help us compare the orthosis influence on the mandibular position. For a physiological rest position the data are not significant. This makes sense because the night-guard does not have any influence on the mandibular position when there is no dental contacts.

\section{Wilcoxon test's results for the teeth in occlusion on the night} guard.

The test will help us compare the orthosis influence with teeth in occlusion on the orthosis. There is a significant difference only for an area with a $\mathrm{p}$ lower than 0.05 . The AmpX/AmpT ration is close from 0.05 and $p$ has a 0.07 value.

\section{Cohen's test results}

The Cohen's D will allow us to calculate the size of the effect. Cohen considers that the effect is weak for a $\mathrm{D}$ lower than 0.2 , average for a $\mathrm{D}$ value at 0.5 and strong for a $0.8 \mathrm{D}$ value. The Cohen's $\mathrm{D}$ value is 0,30087808 . The orthosis impact on the sustentation polygon's area is real but very moderate.

\section{Discussion}

This study is based on a selected population that were selected by having both postural troubles and occlusal pathologies at the same time. The disocclusion diagnosis is made by a clinical exam and then using complementary exams such as electronic recordings of mandibular movements and surface electromyography of masticatory muscles. For the postural troubles, the diagnosis was made by a sport physician or an osteopath but without specifying the type and the intensity of the postural issue. The study shows that for pathological subjects, the statokinesigram and stabilogram set of values analyzed are included in the normal range values given by the stabilometric standard parameters [15]. We can now say that the values are not precise enough from the postural point and therefore doesn't help in making a difference between a patient and a « healthy » subject, hence not allowing us to make comparisons in distinguishing a pathological patient or a healthy subject.

The second hypothesis of our study concerns the impact of occlusal treatment of the values obtained by stabilometric exams before and after an orthosis nightguard. Knowing that the patients included in the study were feeling an improvement of their symptoms (on the Von Korff scale) we can notice that the only parameter that can be adopted is the sustentation polygon's area $(\mathrm{p}<0.05)$; however, the impact on this value is an average and does not seem to be directly related with patients feelings of improvement. The other postural system portals can influence the posture when the record is made but we tried to lower this impact by making the records with closed eyes. We could also have made a difference in high located pains (cervicalgia brachialgia, painful shoulder) and lower located pains (gonalgias, coxalgias, lombalgias).

\section{Conclusion}

The aim of this study was to know if the usage of a stabilometric platform in the occluso-postural treatment issue was useful. As far as the postural deficiency diagnosis is concerned, we can conclude that this analysis is very limited if we want to compare this values to stabilometric standards parameters. The benefit is to compare the different sets of plots for the same patients when for example the eyes are closed or opened. We can also compare the set of plots when the dental occlusion is treated (before and after treatment) only if we restrict it to the sustentation area.

Nevertheless, as the patients' feeling seems to improve (via the Von Korff scale) when it comes to the comparison of the data plots before and after treatment there is no significant difference on the sustentation polygon's area whether the stabilometry or stratokinesigram is concerned. The treatment impact of this value is average but real.

In conclusion, we think that the stabilometric platform as a diagnosis tool is interesting in the posture study when we consider the occlusal aftereffects on the sustentation polygon's area. Complementary studies on the impact of occlusion on the stabilometric records need to be realized considering the influence of the ocular sensor. It would be relevant to do repetitive exams on more healthy subjects by making a difference by age and sex to identify if there is any difference between sex or age group that could become a standard value.

\section{Equipment}

K7 Evaluation System - Myotronics ${ }^{\circledast}$

SATEL ${ }^{\bullet}$ Plateform.

\section{Conflict of interest}

No conflict of interest between authors and Myotronics ${ }^{\oplus}$.

\section{References}

1. Solow B, Sonnesen L (1998) Head posture and malocclusions. Eur J Orthod 20: 685693. [Crossref]

2. Perinetti G (2006) Dental occlusion and body posture: no detectable correlation. Gait Posture 24: 165-168. [Crossref]

3. Manfredini D, Castroflorio T, Perinetti G, Guarda-Nardini L (2012) Dental occlusion, body posture and temporomandibular disorders: where we are now and where we are heading for. J Oral Rehabil 39: 463 471. [Crossref] 
4. Perinetti G (2007) Temporomandibular disorders do not correlate with detectable alterations in body posture. $J$ Contemp Dent Pract 8: 60-67. [Crossref]

5. Perinetti G, Contardo L (2009) Posturography as a diagnostic aid in dentistry: a systematic review. J Oral Rehabil 36: 922-936. [Crossref]

6. Bracco P, Deregibus A, Piscetta R (2004) Effects of different jaw relations on postural stability in human subjects. Neurosci Lett 356: 228-230. [Crossref]

7. Alwarawreh AM, Sarayreh SA, Rabadi HF, Shtaiwi Albdour EA, Al-Marzouq M (2014) Effect of Body Posture on Malocclusion. Pak Oral Dent J 34: 635-639.

8. van't Spijker A, Creugers NH, Bronkhorst EM, Kreulen CM (2011) Body position and occlusal contacts in lateral excursions: a pilot study. Int J Prosthodont 24: 133-136. [Crossref]

9. Wakano S, Takeda T, Nakajima K, Kurokawa K, Ishigami K (2011) Effect of experimental horizontal mandibular deviation on dynamic balance. J Prosthodont Res 55: 228-233. [Crossref]

10. Solow B, Sonnesen L (1998) Head posture and malocclusions. Eur J Orthod 20: 685693. [Crossref]

11. Ohlendorf D, Seebach K, Hoerzer S, Nigg S, Kopp S (2014) The effects of a temporarily manipulated dental occlusion on the position of the spine: a comparison during standing and walking. Spine $J$ 14: 2384-2391. [Crossref]
12. Miles TS, Flavel SC, Nordstrom MA (2004) Control of human mandibular posture during locomotion. J Physiol 554: 216-226. [Crossref]

13. Baldini A (2010) Clinical and instrumental treatment of a patient with dysfunction of the stomatognathic system: a case report. Ann Stomatol (Roma) 1: 2-5. [Crossref]

14. Von Korff M, Ormel J, Keefe FJ, Dworkin SF (1992) Grading the severity of chronic pain. Pain 50: 133-149. [Crossref]

15. Cooper BC, Kleinberg I (2008) Establishment of a temporomandibular physiological state with neuromuscular orthosis treatment affects reduction of TMD symptoms in 313 patients. Cranio 26: 104-117. [Crossref]

16. Esclassan R, Rumerio A, Montsarrat P, Combadazou JC, Champion J, et al. (2016) Optimal duration of ultra low frequency-transcutaneous electrical nerve stimulation (ULF-TENS) therapy for muscular relaxation in neuromuscular occlusion: A preliminary clinical study. Cranio 35: 175-179. [Crossref]

17. Manfredini D, Chiappe G, Bosco M (2006) Research diagnostic criteria for temporomandibular disorders (RDC/TMD) axix I diagnoses in a Italian patient population. J Oral Rehabil 33: 551-558. [Crossref]

18. Gagey P, Weber B (2010) Study of intra-subject random variations of stabilometric Parameters. Med Biol Eng Comput 48: 833-835. [Crossref]

Copyright: $@ 2019$ Combadazou J. This is an open-access article distributed under the terms of the Creative Commons Attribution License, which permits unrestricted use, distribution, and reproduction in any medium, provided the original author and source are credited. 